PROCEEDINGS OF THE

AMERICAN MATHEMATICAL SOCIETY

Volume 88. Number 4. August 1983

\title{
INFINITE-DIMENSIONAL JACOBI MATRICES ASSOCIATED WITH JULIA SETS
}

\author{
M. F. BARNSLEY ${ }^{1}$, J. S. GERONIMO ${ }^{2}$ AND A. N. HARRINGTON
}

\begin{abstract}
Let $B$ be the Julia set associated with the polynomial $T z=z^{1}+k_{1} z^{1-1}$ $+\cdots+k_{1}$, and let $\mu$ be the balanced $T$-invariant measure on $B$. Assuming $B$ is totally real, we give relations among the entries in the infinite-dimensional Jacobi matrix $J$ whose spectral measure is $\mu$. The specific example $T z=z^{3}-\lambda z$ is given. and some of the asymptotic properties of the entries in $J$ are presented.
\end{abstract}

1. Introduction. Let $C$ be the complex plane and $T: C \rightarrow C$ a polynomial, $T(z)=z^{N}+k_{1} z^{N-1}+\cdots+k_{N}$ where $N \geqslant 2$ and each $k_{i} \in C$. Define $T^{0}(z)=z$ and $T^{n}(z)=T \circ T^{n-1}(z)$ for $n \in\{1,2,3, \ldots\}$. A fundamental role in the study of the sequence of iterates $\left\{T^{n}(z)\right\}$ is played by the Julia set $B$. B is the set of points $z \in C$ where $\left\{T^{n}(z)\right\}$ is not normal in the sense of Montel, and a general exposition can be found in Julia [8], Fatou [6,7] and Brolin [5]. It has positive logarithmic capacity, and on it can be placed an equilibrium charge distribution $\mu$. This provides a measure on $B$ which is invariant under $T: B \rightarrow B$ and is such that the system $(B, \mu, T)$ is strongly mixing.

In an earlier paper [1] we investigated general properties of $\mu$ and its associated orthogonal monic polynomials. Here we restrict attention to the case where $B$ is a compact subset of the real line, and the orthogonal polynomials satisfy a three-term recurrence formula. In [2] we proved, for $N=2$, relationships connecting the coefficients, which permit all the polynomials to be calculated in a recursive fashion. Here we generalized the relationships so that the orthogonal polynomials of all degrees can be obtained for any $T$ for which $B$ is a compact subset of the real line (Theorem 1). The results are illustrated for $T(z)=z^{3}-\lambda z$ with $\lambda \geqslant 3$. When $\lambda=3$ the polynomials are those of Chebychev, shifted to the interval $[-2,2]$, and when $\lambda>3$ they become a generalization whose support is a Cantor set. In this case we establish that both the coefficients (Theorem 2) and the associated Jacobi matrix $J$ (Theorem 3) display an asymptotic self-reproducing property.

\section{Preliminaries.}

Definition 1. $\mu$ is a balanced $T$-invariant Borel measure on $B$ if $\mu$ is a probability measure supported on $B$, such that for any complete assignment of branches of $T^{-1}$, namely $T_{j}^{-1}$ for $j \in\{1,2,3, \ldots, N\}, \mu\left(T_{j}^{-1}(S)\right)=\mu(S) / N$ for each Borel set $S$.

Received by the editors March 23, 1982.

1980 Mathematics Subject Classification. Primary 30C10; Secondary 47B25.

'Supported by NSF grant MCS- 8104862 .

${ }^{2}$ Supported by NSF grant MCS- 8002731 . 
There is only one balanced $T$-invariant measure on $B$, and the equilibrium measure of Brolin is balanced [3]. If $\mu$ is balanced and $f \in L^{1}(B, \mu)$, then [1]

$$
\left\langle z^{j} f(T(z))\right\rangle=S_{j}\langle f(z)\rangle / N \text { for } j \in\{1,2, \ldots, N-1\},
$$

where $\langle f(z)\rangle=\int_{B} f(z) d \mu(z)$. Here

$$
S_{j}=-j k_{j}-\sum_{l=1}^{j-1} k_{l} S_{l}
$$

with $k$, the coefficient of $Z^{N-l}$ in $T$ for $l \in\{1,2, \ldots, N\}$.

In [1] we showed that the sequence of monic polynomials $\left\{P_{n}(z)\right\}_{n=0}^{\infty}$, orthogonal with respect to $\mu$ according to $\left\langle\overline{P_{l}(z)} P_{m}(z)\right\rangle=0$ for $l \neq m$, obey the following relations:

(a) $P_{1}(z)=z+k_{1} / N$

(b) $P_{l N}(z)=P_{l}(T(z))$ for $l \in\{0,1,2, \ldots\}$,

(c) $P_{N^{\prime}}(z)=T^{\prime}(z)+k_{1} / N$ for $l \in\{0,1,2, \ldots\}$.

3. Results. When $B$ is a subset of the real line the orthonormal polynomials with respect to $\mu$ obey (b) and the following relation.

(3) $a(n+1) p_{n+1}(x)+b(n) p_{n}(x)+a(n) p_{n-1}(x)=x p_{n}(x), \quad n \in\{0,1,2, \ldots\}$,

$$
p_{-1}(x)=0, \quad p_{0}(x)=1 \text {, }
$$

where

$$
a(n)=\left\langle x p_{n} p_{n-1}\right\rangle \text { for } n \in\{1,2,3, \ldots\}
$$

and

$$
b(n)=\left\langle x p_{n}^{2}\right\rangle \text { for } n \in\{0,1,2, \ldots\} .
$$

The recurrence formula (3) can be recast as the formal operator equation

$$
J \psi=x \psi
$$

where

$$
J=\left(\begin{array}{cccc}
b(0) & a(1) & 0 & \ldots \\
a(1) & b(1) & a(2) & \ldots \\
0 & a(2) & b(2) & \ldots \\
\cdot & \cdot & . & \ldots \\
. & . & . & \ldots \\
. & . & . & \ldots
\end{array}\right)
$$

and $\psi^{T}=\left(p_{0}, p_{1}, p_{2}, \ldots\right) . J$ can be treated as a selfadjoint operator acting on $l_{2}$. In [2] we showed that the coefficients in $J$ obey certain recurrence formulas when $T$ is quadratic; see also [4]. We generalize that result here.

Proceeding formally we have

$$
J^{l} \psi=x^{l} \psi \text { for } l \in\{0,1,2, \ldots\},
$$

which leads to

$$
\left\langle p_{n N} J^{l} \psi \hat{e}_{n N+1}\right\rangle=\left\langle x^{l} p_{n N}^{2}\right\rangle
$$


for $n \in\{0,1,2, \ldots\}$, where $\hat{e}_{k}$ is the $l_{2}$ vector with one in the $k$ th place and zeros elsewhere. Observe also that the invariance of $\mu$ together with (b) implies

$$
\begin{aligned}
a(n) & =\left\langle x p_{n} p_{n-1}\right\rangle=\left\langle T(x) p_{n N} p_{n N-N}\right\rangle \\
& =a(n N) a(n N-1) \cdots a(n N-N+1), \quad n \in\{1,2,3, \ldots\} .
\end{aligned}
$$

THEOREM 1. Let $a(n)=b(n-1)=0$ for $n \leqslant 0$. Then all of the coefficients in $J$ can be calculated recursively using (8) and (7) with $l \in\{1,2, \ldots, 2 N-1\}$.

The proof will require two lemmas.

LEMMA 1. Let $\left\{p_{n}\right\}_{0}^{\infty}$ be the orthonormal polynomials associated with the balanced $T$-invariant $\mu$. Then

$$
\left\langle x^{l} p_{n N}^{2}\right\rangle=D(l) \text { for } l \in\{1,2, \ldots, 2 N-1\},
$$

where

$$
D(l)= \begin{cases}N^{-1} S_{l} & \text { when } l \in\{1,2, \ldots, N-1\}, \\ N^{-1} S_{l-N} b(n)-\sum_{j=1}^{N} k_{j} D(l-j) & \text { when } l \in\{N, \ldots, 2 N-1\},\end{cases}
$$

where $S_{0}=N$ and $S_{l}$ is otherwise as defined in (2).

Proof of Lemma 1. For $l \in\{1,2, \ldots, N-1\}$ the result follows from (1) with $f=p_{n N}^{2}$. For $l=N+m$,

$$
x^{N+m}=x^{m} T(x)-\sum_{j=1}^{N} k_{j} x^{m+N-j} .
$$

The lemma now follows on multiplying through by $p_{n N}^{2}$, integrating, and using the fact that

$$
\left\langle x^{m} T(x) p_{n N}^{2}\right\rangle=N^{-1} S_{m}\left\langle x p_{n}^{2}\right\rangle=N^{-1} S_{m} b(n)
$$

for $m \in\{0,1,2, \ldots, N-1\}$.

One can now see that the dependence on $n$ on the right-hand side enters only through $b(n)$.

LEMMA 2. Let $C^{\prime}(n N+1, n N+1)$ denote the $(n N+1, n N+1)$ entry in $J^{l}$. When $l=2 k$, the coefficient in $C^{2 k}(n N+1, n N+1)$ with the highest index is $a(n N+k)$ and all other coefficients have lower indices. When $l=2 k+1$, the coefficients in $C^{2 k+1}(n N+1, n N+1)$ with the highest index are $a(n N+k)$ and $b(n N+k)$; all other coefficients have lower indices.

Proof of Lemma 2. We begin by computing $C^{l}(n N+1, n N+1)$ with the aid of (7). Thus

$$
\begin{array}{r}
C^{l}(n N+1, n N+1)=a(n N) C^{l-1}(n N, n N+1)+b(n N) C^{l-1}(n N+1, n N+1) \\
+a(n N+1) C^{l-1}(n N+2, n N+1), \quad l \in\{1,2, \ldots, 2 N-1\},
\end{array}
$$

with

$$
C^{1}(i, j)=a(i-1) \delta_{i-1, j}+b(i-1) \delta_{i, j}+a(i) \delta_{i+1, j},
$$


and

$$
\begin{aligned}
C^{m}(i, j)= & a(i-1) C^{m-1}(i-1, j) b(i-1) \\
& +C^{m-1}(i, j)+a(i) C^{m-1}(i+1, j) .
\end{aligned}
$$

It follows immediately from (14) that $C^{m}(i, j)=0$ if $|i-j|>m$. From (13) and (14) we find

$$
C^{1}(n N+1, n N+1)=b(n N)
$$

and

$$
C^{2}(n N+1, n N+1)=a(n N)^{2}+b(n N)^{2}+a(n N+1)^{2} .
$$

Let us now assume that the lemma holds up to $2 k-1$. Then

$$
\begin{aligned}
& C^{2 k}(n N+1, n N+1)=a(n N+1) C^{2 k-1}(n N+2, n N+1) \\
& \quad+b(n N) C^{2 k-1}(n N+1, n N+1)+a(n N) C^{2 k-1}(n N+1, n N+1) .
\end{aligned}
$$

One can easily show by induction that if $a(l)$ or $b(n)$ appear in $C^{m}(i, j)$ then $l \leqslant(m+i+j) / 2$ and $n \leqslant(m+i+j-1) / 2$. Consequently one need only consider the first term on the right-hand side of (17). Therefore

$$
\begin{aligned}
C^{2 k}(n N+1, n N+1)=\left[\prod_{l=1}^{k} a(n N+l)\right] C^{k}(n N+k+1, n N+1) \\
+\{\text { terms containing only coefficients with indices lower than } n N+k\} .
\end{aligned}
$$

But from (14) we have

$$
C^{k}(n N+k+1, n N+1)=\prod_{l=1}^{k} a(n N+l),
$$

whence

$$
\begin{aligned}
C^{2 k}(n N+1, & n N+1)=\left[\prod_{l=1}^{k} a(n N+l)\right]^{2} \\
& +\{\text { terms involving only coefficients with indices lower than } n N+k\} .
\end{aligned}
$$

Likewise,

$$
\begin{gathered}
C^{2 k+1}(n N+1, n N+1)=\left[\prod_{l=1}^{k} a(n N+l)\right] C^{k+1}(n N+k+1, n N+1) \\
+\{\text { terms involving only } a(l) \text { and } b(l-1) \text { with } l<n N+k\}
\end{gathered}
$$

and (14) now yields

$$
\begin{aligned}
C^{2 k+1}(n N+1, n N+1)=\left[\prod_{l=1}^{k} a(n N+l)\right]^{2} b(n N+k) \\
+\{\text { terms involving only } a(l) \text { and } b(l-1) \text { with } l<n N+k\} .
\end{aligned}
$$

This completes the proof of Lemma 2. 
Proof of Theorem 1. If one is given $a(i)$ and $b(i)$ for $i<N n$, then Lemmas 1 and 2 , together with (8), provide $2 N$ relations from which one can explicitly calculate $a(n N+l)$ and $b(n N+l)$ for $l \in\{0,1,2, \ldots, N-1\}$. This completes the proof.

COROLlary 1. If $B$ is an interval on the real line then $B=[a, b]$ with $a=-k_{1} / N$ -2 and $b=-k_{1} / N+2$. Moreover, $d \mu=d x / \pi\{(b-x)(x-a)\}^{1 / 2}$, and $T(x)+$ $k_{1} / N$ is the monic Chebychev polynomial of degree $N$ on $B$.

Proof. If $B$ is an interval then the electrical equilibrium distribution $\mu$ is just the measure associated with the Chebychev polynomials of the first kind. Since all the off-diagonal entries in $J$ except for $a(1)$ are the same, (6) implies these must equal unity. Likewise, all diagonal entries in $J$ must be equal to $-k_{1} / N$, and the proof is completed.

4. An example. We examine the case $T(z)=z^{3}-\lambda z$ with $\lambda \geqslant 3$, for which Theorem 1 yields

$$
\begin{gathered}
b(n)=0, \\
a(3 n+1)^{2}=2 \lambda / 3-a(3 n)^{2}, \\
a(3 n+2)^{2}=\lambda / 3
\end{gathered}
$$

and

$$
a(3 n) a(3 n-1) a(3 n-2)=a(n) .
$$

From these relations and Corollary 1 it is easy to see that $B=[-2,2]$ when $\lambda=3$. For $\lambda>3$ it follows from [5] that $B$ is a totally disconnected perfect subset of the real line, with Lebesgue measure zero. As such, it is a generalized Cantor set.

Lemma 3. For $\lambda>3$ and $n \in\{1,2,3, \ldots\}, 0<a(3 n)<1$ and $a(3 n)<a(n)$.

Proof. From (23) and (25) it follows that $a(1)^{2}=2 \lambda / 3$ and $a(3)^{2}=3 / \lambda$. Furthermore, from (23)-(25) we have

$$
a(3 n)^{2}=\frac{3}{\lambda} \frac{a(n)^{2}}{2 \lambda / 3-a(3 n-3)^{2}},
$$

and the lemma follows by induction and equations (23) and (24).

TheOREM 2. For $\lambda>3$ and $m, s \in\{0,1,2, \ldots\}$,

$$
\operatorname{Lim}_{n \rightarrow \infty} a\left(m 3^{n}+s\right)^{2}=a(s)^{2} \text {. }
$$

Proof. First consider the case $s=0$. Then from (26)

$$
\begin{aligned}
a\left(m 3^{n}\right)^{2} & =(3 / \lambda) a\left(m 3^{n-1}\right)^{2} /\left(2 \lambda / 3-a\left(m 3^{n}-3\right)^{2}\right) \\
& <(3 / \lambda) a\left(m 3^{n-1}\right)^{2} /(2 \lambda / 3-1)<(3 / \lambda)^{n}(2 \lambda / 3-1)^{-n} a(m)^{2} .
\end{aligned}
$$

Because $3 / \lambda<1$, and $2 \lambda / 3-1>1$, for $\lambda>3$ we now have $\operatorname{Lim}_{n \rightarrow \infty} a\left(m 3^{n}\right)^{2}=0$. The proof is now completed by induction on $m$ for $s=3 m+k, k \in\{0,1,2, \ldots\}$, using (23)-(25). 
Results similar to Lemma 3 and Theorem 2 are valid for $T(z)=(z-\lambda)^{2}$ with $\lambda \geqslant 2$ and follow from [2]; see, for example, [4].

Now consider the sequence of infinite-dimensional Jacobi matrices $\left\{J^{\left(m 3^{n}\right)}\right\}$ defined for $m, n \in\{0,1,2, \ldots\}$ by

$$
J^{\left(m 3^{n}\right)}=\left(\begin{array}{cccc}
0 & a\left(m 3^{n}+1\right) & 0 & . \\
a\left(m 3^{n}+1\right) & 0 & a\left(m 3^{n}+2\right) & . \\
0 & a\left(m 3^{n}+2\right) & 0 & . \\
. & . & . & .
\end{array}\right) .
$$

Here the coefficients $a(i)$ are those determined by (23)-(25). Since the support $B$ of the spectral measure of $J$ is compact, it also is for each $J^{\left(m 3^{n}\right)}$, and, hence, each matrix corresponds to a selfadjoint operator in $l_{2}$.

TheOREM 3. For each $m \in\{0,1,2, \ldots\}$ and $\lambda \geqslant 3$ the sequence of operators $\left\{J^{\left(m 3^{n}\right)}\right\}_{n=0}^{\infty}$ converges strongly to $J$.

This theorem, and indeed Theorem 2 also, are immediate when $\lambda=3$ because then

$$
J=\left(\begin{array}{lllll}
0 & 1 & 0 & 0 & \cdot \\
1 & 0 & 1 & 0 & \cdot \\
0 & 1 & 0 & 1 & \cdot \\
0 & 0 & 1 & 0 & \cdot \\
\cdot & \cdot & \cdot & \cdot & .
\end{array}\right)
$$

Proof of TheOREM 3. Since the spectrum of $J$ is compact, the entries of $J^{\left(m 3^{n}\right)}$ are uniformly bounded. The result now follows since the weak convergence implied by Theorem 2 implies the strong operator convergence

$$
\lim _{n \rightarrow \infty}\left\|\left(J-J^{\left(m 3^{n}\right)}\right) x\right\|=0, \text { for all } x \in l_{2},
$$

for banded matrices. This completes the proof.

\section{REFERENCES}

1. M. F. Barnsley, J. S. Geronimo and A. N. Harrington, Orthogonal polynomials associated with invariant measures on Julia sets, Bull. Amer. Math. Soc. (N.S.) 7 (1982), 381-384.

2. __ On the invariant sets of a family of quadratic maps, Comm. Math. Phys. (to appear).

3. Geometry, electrostatic measure, and orthogonal polynomials on Julia sets for polynomials, Ergod. Th. \& Dynam. Sys. (submitted).

4. D. Bessis, M. L. Mehta and P. Moussa, Orthogonal polynomials on a family of Cantor sets and the problem of iterations of quadratic mappings, Lett. Math. Phys. 6 (1982), 123-140.

5. H. Brolin, Invariant sets under iteration of rational functions, Ark. Mat. 6 (1965), 103-144.

6. P. Fatou, Sur les equations fonctionelles, Bull. Soc. Math. France 47 (1919), 161-271.

7. Sur les equations fonctionelles, Bull. Soc. Math. France 48 (1920), 33-94, 208-314.

8. G. Julia, Mémoire sur l'iteration des fonctions rationelles, J. Math. Pures Appl. 1 (1918), 47-245.

School of Mathematics, Georgia Institute of Technology, Atlanta, Georgia 30332 